\title{
Text-mining applied to autoimmune disease research: the Sjögren's syndrome knowledge base
}

\author{
Sven-Ulrik Gorr ${ }^{1 *}$, Trevor J Wennblom², Steve Horvath ${ }^{3}$, David TW Wong ${ }^{4}$ and Sara A Michie ${ }^{5}$
}

\begin{abstract}
Background: Sjögren's syndrome is a tissue-specific autoimmune disease that affects exocrine tissues, especially salivary glands and lacrimal glands. Despite a large body of evidence gathered over the past 60 years, significant gaps still exist in our understanding of Sjögren's syndrome. The goal of this study was to develop a database that collects and organizes gene and protein expression data from the existing literature for comparative analysis with future gene expression and proteomic studies of Sjögren's syndrome.

Description: To catalog the existing knowledge in the field, we used text mining to generate the Sjögren's Syndrome Knowledge Base (SSKB) of published gene/protein data, which were extracted from PubMed using text mining of over 7,700 abstracts and listing approximately 500 potential genes/proteins. The raw data were manually evaluated to remove duplicates and false-positives and assign gene names. The data base was manually curated to 477 entries, including 377 potential functional genes, which were used for enrichment and pathway analysis using gene ontology and KEGG pathway analysis.
\end{abstract}

Conclusions: The Sjögren's syndrome knowledge base (http://sskb.umn.edu) can form the foundation for an informed search of existing knowledge in the field as new potential therapeutic targets are identified by conventional or high throughput experimental techniques.

\section{Background}

Sjögren's syndrome is a tissue-specific autoimmune disease that affects exocrine tissues, especially salivary glands and lacrimal glands. It is one of the most common autoimmune disorders in the U.S., with an estimated prevalence of 2-4 million people. The autoimmunemediated damage of the salivary and lacrimal glands in Sjögren's syndrome leads to a decrease in the production of saliva and tears and to the development of dry mouth and dry eyes. Without the lubricating and protective functions of saliva and tears, the oral and ocular surfaces are subject to infections and discomfort, leading to a significantly reduced quality of life $[1,2]$.

Development of Sjögren's syndrome requires a complex interplay between a number of genetic, hormonal and environmental factors, most of which have not been defined. Genetic linkages, especially involving major

\footnotetext{
* Correspondence: sugorr@umn.edu

${ }^{1}$ Department of Diagnostic and Biological Sciences, University of Minnesota

School of Dentistry, Minneapolis, MN 55455, USA

Full list of author information is available at the end of the article
}

histocompatibility complex (MHC) genes, have been reported for Sjögren's syndrome but it is not clear if, or how, the associated genes are involved in the development of the disease [3]. Additional non-MHC genes have also been linked with the development of Sjögren's syndrome.

In addition to genetic predisposition, some studies suggest that infection of a genetically-susceptible individual by a virus or other pathogen might trigger the development of an autoimmune disease [4]. The proposed mechanisms include activation of the innate immune system, release of self antigens from damaged or apoptotic tissues, and molecular mimicry that results in activation of $\mathrm{T}$ cells and/or $\mathrm{B}$ cells that react with tissue antigens [4].

Both the innate and the adaptive immune systems are involved in the pathogenesis of Sjögren's syndrome. The type I interferon (IFN) pathway, which plays an important role in the innate immune response to viruses, is also thought to play an important role in the development of Sjögren's syndrome and other autoimmune disorders, including SLE $[5,6]$. Moreover, type I IFNs can

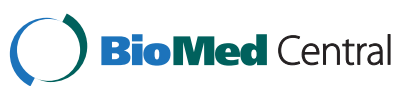


activate the adaptive immune system directly, by binding to IFN receptors on antigen presenting cells, $\mathrm{T}$ cells and B cells, or indirectly, by inducing the production and release of cytokines and chemokines that bind to these cells.

Autoantibodies to intracellular antigens, notably the nuclear proteins SSA/Ro and SSB/La, are found in the sera of many patients with Sjögren's syndrome. These autoantibodies are thought to develop when intracellular antigens, some of which have undergone proteolytic cleavage that reveals new antigenic epitopes, become "visible" to the immune system in membrane blebs on the surface of apoptotic cells [7]. Alternatively, antigenic epitopes from bacteria and viruses, including EpsteinBarr virus (EBV) and coxsackie virus, may act as molecular mimics that trigger the development of antibodies that cross react with similar epitopes on target tissue autoantigens $[2,8,9]$. Although autoantibodies to intracellular antigens are useful in the diagnosis of Sjögren's syndrome, it is not clear if they play a direct role in the development of salivary gland and lacrimal gland damage and hypofunction. In contrast, autoantibodies to the M3 muscarinic acetylcholine receptor (M3R) have been directly implicated in salivary gland hypofunction in the nonobese diabetic (NOD) mouse model of Sjögren's syndrome [10]. Importantly, functioninhibiting anti-M3R autoantibodies are found in the sera of many patients with Sjögren's syndrome [11].

Current therapy for Sjögren's syndrome usually consists of palliative treatment that relieves the symptoms of dry eye and dry mouth, but fails to modify the underlying disease. Novel disease-modifying treatment strategies, based on recent immunological insights in Sjögren's syndrome and other autoimmune diseases, have met with mixed results [12]. For example, in recent clinical trials, treatment of Sjögren's syndrome patients with a B cell-depleting anti-CD20 monoclonal antibody (rituximab) led to significant improvement of the stimulated whole saliva flow rate and a reduction in parotid gland inflammation [13]. In contrast, TNF $\alpha$ inhibitors have been ineffective in the treatment of Sjögren's syndrome. Detailed studies on the immune response in Sjögren's syndrome patients treated with one of the inhibitors (etanercept) revealed an increase in the circulating levels of TNF $\alpha$ [14]. These results suggest that TNF $\alpha$ may not play a pivotal role in the disease and that other therapeutic targets must be identified.

Despite a large body of evidence gathered over the past 60 years, significant gaps still exist in our understanding of Sjögren's syndrome. Recent gene expression and proteomic studies have identified many genes and pathways that may play a role in the pathogenesis of Sjögren's syndrome [15-17]. However, validation of these data will require significant additional effort. As an initial step in this validation, we have compiled the published data on Sjögren's syndrome that is not derived from gene expression or proteomic studies. No such unifying database currently exists. Through data curation, the existing data have been uniformly formatted to allow systematic retrieval and comparisons to newly generated gene expression data. As an example of its functionality, the Sjögren's Syndrome Knowledge Base (SSKB) was analyzed for biological functions and pathways that are likely to play a role in the disease.

\section{Construction and content Data mining}

To catalog the existing knowledge in the field, we used text mining to generate the Sjögren's Syndrome Knowledge Base (SSKB) of published gene/protein data (http://sskb.umn.edu/) [18]. The focus of this data-base is on individually identified genes and proteins. Thus, microarray experiments were not included. The raw data for SSKB was extracted from PubMed [19]) using the text mining program EBIMed (http://www.ebi.ac.uk/ Rebholz-srv/ebimed/) [20] with the search term "Sjogren's Syndrome" restricted to "MeshHeadingsList". The foundational search identified over 7,700 abstracts and approximately 500 potential genes/proteins. The SSKB is continually updated by regular automated searches of PubMed followed by manual curation.

\section{Curation of raw data}

The identified abstracts were manually evaluated to remove duplicates and false-positives. In older publications, where gene names were not readily identifiable, names were assigned based on in depth evaluation of the protein name context and available gene data in public databases, including the National Center for Biotechnology Information's Entrez search engine [21] and UniProt [22,23]. The SSKB includes data from human studies and animal models. For the genes identified in animal models, the human homolog was identified by automated ortholog search, using WebGestalt 2.0 $[24,25]$. These steps reduced the database to 477 current entries. The online database contains the fully curated data and currently contains 413 entries, which can be accessed at http://sskb.umn.edu. Updates and newly curated data are continually added.

The 477 entries were sorted to identify autoantigens and viral/bacterial antigens, resulting in 377 potential functional genes, which were used for enrichment and pathway analysis.

\section{Enrichment analysis}

The 377 human gene entries were used for subsequent enrichment analyses in Webgestalt [24,25]. Gene enrichment in the SSKB gene set was compared to the human 
genome using the hypergeometric test with multiple test adjustment [26] and a significance level of $\mathrm{P}<0.01$.

The Gene Ontology [27,28] was accessed with Webgestalt and analysis was restricted to processes and functions represented by two or more genes. Pathway analysis was performed with Webgestalt in the Kyoto Encyclopedia of Genes and Genomes (KEGG) [29,30]. The selection was restricted to pathways with 4 or more genes represented, resulting in identification of 72 KEGG pathways. The "salivary secretion" pathway (KO04970) was recently added to KEGG (11/9/10) and was not included in this analysis. This pathway contains 59 genes, seven of which are found in the SSKB gene set.

\section{Utility and discussion}

We constructed a database containing proteins and genes associated with Sjögren's syndrome in human disease or animal models, as identified by text mining of published data. The public SSKB currently contains 413 genes/proteins and can be viewed online (http://sskb. umn.edu/). All genes have been assigned gene symbols and UniProt IDs, which allows rapid retrieval of genespecific data from external databases. The SSKB data base can be used to determine whether a list of genes is enriched with known Sjögren's syndrome genes and one can carry out a function enrichment analysis (hypergeometric distribution). Individual genes and the corresponding gene products, synonyms and alternate names can be searched by using a web browser search function. Autoantigens, viral antigens and bacterial antigens are separately identified under "Antigens". The SSKB is continually maintained and updated and new genes are added as their analysis is completed.

Based on the abstracts used to retrieve the SSKB genes/proteins, 85 proteins were initially characterized as autoantigens and 15 proteins were characterized as viral (14) or bacterial (1) antigens. Not surprisingly, SSA/Ro and SSB/La were among the most frequently retrieved autoantigens. It has been proposed that viral or bacterial antigens act as autoimmune triggers by molecular mimicry of endogenous human proteins $[2,8,9]$. However, eight of the 14 putative viral antigens in SSKB were selected for BLAST analysis, which did not identify strong sequence similarity with human proteins (not shown).

The 377 proteins not identified as autoantigens or microbial antigens were considered candidates for functional genes that could play a role in the initiation and progression of Sjögren's syndrome. Since the gene list contains data from humans and animals, the corresponding human genes were identified, with the assumption that genes identified in animal models of Sjögren's syndrome may also be involved in the human disease.

\section{Gene ontology}

The Gene Ontology database [27] was queried to identify the biological processes, cellular components and molecular functions associated with genes in the SSKB (Table 1). The 40 most highly enriched entries were identified in each category.

The most highly enriched biological processes (19 of 40 ; 18 of the top 20) were associated with immune function, including leukocyte proliferation, leukocyte activation, and regulation of the immune response. Other prominent biological processes were associated with apoptosis and cell death. Thus, the SSKB data set is consistent with recent microarray data [16] and reflects current models for the biological processes involved in the pathogenesis of Sjögren's syndrome [5,31,32].

The most highly enriched cellular component was the calcineurin complex, which plays a major role in the activation of $\mathrm{T}$ cells. Interestingly, in placebo-controlled clinical trials, treatment of Sjögren's syndrome patients with eye drops that contain the calcineurin inhibitor cyclosporine, led to significant improvement in several of the signs and symptoms of dry eye [33].

Other highly enriched cellular components include: 1) platelet alpha granules. Although platelet activation has been reported in the salivary glands of Sjögren's syndrome patients [34], a direct search of PubMed for "platelet alpha granules" with "sjogren's" did not retrieve any published studies. Thus, while the proteins identified were retrieved from the literature, their potential association with platelet alpha granules in Sjögren's syndrome has not previously been noted. 2) MHC protein complexes were identified and are presumably involved in the presentation of autoantigens [16]. 3) The finding that protein-lipid complexes and lipoprotein particles are associated with Sjögren's syndrome may be consistent with changes in serum lipid levels in Sjögren's syndrome patients [35] although the prevalence of anti-phospholipid antibodies is low in Sjögren's syndrome [36]. 4) Nerve terminals and axons were also prominent cellular components, consistent with the known neurological component of Sjögren's syndrome [37].

In molecular function, nitric oxide synthase (NOS) activity was the most highly enriched, although only three genes (NOS1-3) were identified. Nitric oxide (NO) signaling appears to be directly affected in salivary and lacrimal glands in Sjögren's syndrome [38]. Other highly enriched molecular functions include chemokine and cytokine activity/receptor binding (8 of the top 15) and peptidase activities.

\section{Pathway analysis}

The SSKB gene list was submitted to KEGG [29] to identify biological pathways potentially associated with Sjögren's syndrome. A total of 72 KEGG pathways 
Table 1 Gene Ontology enrichment analysis

\begin{tabular}{|c|c|c|c|c|c|}
\hline Rank & BIOLOGICAL PROCESS & GO ID & Reference Genes & Observed Genes & Ratio \\
\hline 1 & regulation of lymphocyte proliferation & GO:0050670 & 81 & 32 & $39.51 \%$ \\
\hline 2 & regulation of leukocyte proliferation & GO:0070663 & 82 & 32 & $39.02 \%$ \\
\hline 3 & regulation of mononuclear cell proliferation & GO:0032944 & 82 & 32 & $39.02 \%$ \\
\hline 4 & $\begin{array}{l}\text { adaptive immune response based on somatic } \\
\text { recombination of immune receptors built from } \\
\text { immunoglobulin superfamily domains }\end{array}$ & GO:0002460 & 112 & 38 & $33.93 \%$ \\
\hline 5 & adaptive immune response & GO:0002250 & 113 & 38 & $33.63 \%$ \\
\hline 6 & lymphocyte proliferation & GO:0046651 & 112 & 37 & $33.04 \%$ \\
\hline 7 & leukocyte proliferation & GO:0070661 & 114 & 37 & $32.46 \%$ \\
\hline 8 & mononuclear cell proliferation & GO:0032943 & 114 & 37 & $32.46 \%$ \\
\hline 9 & regulation of lymphocyte activation & GO:0051249 & 141 & 42 & $29.79 \%$ \\
\hline 10 & regulation of cell activation & GO:0050865 & 168 & 46 & $27.38 \%$ \\
\hline 11 & regulation of leukocyte activation & GO:0002694 & 159 & 43 & $27.04 \%$ \\
\hline 12 & positive regulation of immune system process & GO:0002684 & 229 & 60 & $26.20 \%$ \\
\hline 13 & regulation of immune response & GO:0050776 & 218 & 54 & $24.77 \%$ \\
\hline 14 & immune effector process & GO:0002252 & 200 & 45 & $22.50 \%$ \\
\hline 15 & regulation of immune system process & GO:0002682 & 362 & 79 & $21.82 \%$ \\
\hline 16 & lymphocyte activation & GO:0046649 & 272 & 59 & $21.69 \%$ \\
\hline 17 & leukocyte activation & GO:0045321 & 324 & 66 & $20.37 \%$ \\
\hline 18 & inflammatory response & GO:0006954 & 359 & 71 & $19.78 \%$ \\
\hline 19 & cell activation & GO:0001775 & 366 & 71 & $19.40 \%$ \\
\hline 20 & immune response & GO:0006955 & 750 & 133 & $17.73 \%$ \\
\hline 21 & regulation of response to stimulus & GO:0048583 & 441 & 75 & $17.01 \%$ \\
\hline 22 & defense response & GO:0006952 & 657 & 100 & $15.22 \%$ \\
\hline 23 & immune system process & GO:0002376 & 1066 & 162 & $15.20 \%$ \\
\hline 24 & response to wounding & GO:0009611 & 560 & 85 & $15.18 \%$ \\
\hline 25 & response to external stimulus & GO:0009605 & 904 & 110 & $12.17 \%$ \\
\hline 26 & multi-organism process & GO:0051704 & 668 & 79 & $11.83 \%$ \\
\hline 27 & regulation of programmed cell death & GO:0043067 & 812 & 92 & $11.33 \%$ \\
\hline 28 & regulation of apoptosis & GO:0042981 & 805 & 91 & $11.30 \%$ \\
\hline 29 & regulation of cell death & GO:0010941 & 815 & 92 & $11.29 \%$ \\
\hline 30 & regulation of cell proliferation & GO:0042127 & 739 & 79 & $10.69 \%$ \\
\hline 31 & apoptosis & GO:0006915 & 1063 & 102 & $9.60 \%$ \\
\hline 32 & programmed cell death & GO:0012501 & 1071 & 102 & $9.52 \%$ \\
\hline 33 & response to chemical stimulus & GO:0042221 & 1243 & 117 & $9.41 \%$ \\
\hline 34 & cell proliferation & GO:0008283 & 1056 & 98 & $9.28 \%$ \\
\hline 35 & death & GO:0016265 & 1171 & 107 & $9.14 \%$ \\
\hline 36 & cell death & GO:0008219 & 1167 & 106 & $9.08 \%$ \\
\hline 37 & response to stress & GO:0006950 & 1696 & 144 & $8.49 \%$ \\
\hline 38 & positive regulation of biological process & GO:0048518 & 1865 & 153 & $8.20 \%$ \\
\hline 39 & positive regulation of cellular process & GO:0048522 & 1699 & 130 & $7.65 \%$ \\
\hline 40 & response to stimulus & GO:0050896 & 3471 & 221 & $6.37 \%$ \\
\hline Rank & CELLULAR COMPONENT & GO ID & Count & Observed & Ratio \\
\hline 1 & calcineurin complex & GO:0005955 & 5 & 3 & $60.00 \%$ \\
\hline 2 & external side of plasma membrane & GO:0009897 & 131 & 40 & $30.53 \%$ \\
\hline
\end{tabular}


Table 1 Gene Ontology enrichment analysis (Continued)

\begin{tabular}{|c|c|c|c|c|c|}
\hline 3 & platelet alpha granule lumen & GO:0031093 & 41 & 12 & $29.27 \%$ \\
\hline 4 & MHC class II protein complex & GO:0042613 & 14 & 4 & $28.57 \%$ \\
\hline 5 & nerve terminal & GO:0043679 & 14 & 4 & $28.57 \%$ \\
\hline 6 & cytoplasmic membrane-bounded vesicle lumen & GO:0060205 & 44 & 12 & $27.27 \%$ \\
\hline 7 & vesicle lumen & GO:0031983 & 46 & 12 & $26.09 \%$ \\
\hline 8 & integrin complex & GO:0008305 & 29 & 7 & $24.14 \%$ \\
\hline 9 & platelet alpha granule & GO:0031091 & 52 & 12 & $23.08 \%$ \\
\hline 10 & high-density lipoprotein particle & GO:0034364 & 24 & 5 & $20.83 \%$ \\
\hline 11 & MHC protein complex & GO:0042611 & 38 & 7 & $18.42 \%$ \\
\hline 12 & plasma lipoprotein particle & GO:0034358 & 34 & 6 & $17.65 \%$ \\
\hline 13 & protein-lipid complex & GO:0032994 & 34 & 6 & $17.65 \%$ \\
\hline 14 & cell surface & GO:0009986 & 305 & 51 & $16.72 \%$ \\
\hline 15 & axon part & GO:0033267 & 48 & 7 & $14.58 \%$ \\
\hline 16 & extracellular space & GO:0005615 & 670 & 84 & $12.54 \%$ \\
\hline 17 & receptor complex & GO:0043235 & 113 & 13 & $11.50 \%$ \\
\hline 18 & secretory granule & GO:0030141 & 174 & 19 & $10.92 \%$ \\
\hline 19 & membrane raft & GO:0045121 & 131 & 14 & $10.69 \%$ \\
\hline 20 & extracellular region part & GO:0044421 & 939 & 94 & $10.01 \%$ \\
\hline 21 & axon & GO:0030424 & 148 & 14 & $9.46 \%$ \\
\hline 22 & cell soma & GO:0043025 & 155 & 13 & $8.39 \%$ \\
\hline 23 & soluble fraction & GO:0005625 & 297 & 24 & $8.08 \%$ \\
\hline 24 & cytoplasmic vesicle part & GO:0044433 & 177 & 13 & $7.34 \%$ \\
\hline 25 & extracellular region & GO:0005576 & 1984 & 143 & $7.21 \%$ \\
\hline 26 & basolateral plasma membrane & GO:0016323 & 190 & 13 & $6.84 \%$ \\
\hline 27 & lysosome & GO:0005764 & 206 & 14 & $6.80 \%$ \\
\hline 28 & integral to plasma membrane & GO:0005887 & 1183 & 72 & $6.09 \%$ \\
\hline 29 & intrinsic to plasma membrane & GO:0031226 & 1206 & 73 & $6.05 \%$ \\
\hline 30 & cytoplasmic membrane-bounded vesicle & GO:0016023 & 537 & 32 & $5.96 \%$ \\
\hline 31 & membrane-bounded vesicle & GO:0031988 & 555 & 32 & $5.77 \%$ \\
\hline 32 & extracellular matrix & GO:0031012 & 335 & 19 & $5.67 \%$ \\
\hline 33 & neuron projection & GO:0043005 & 318 & 18 & $5.66 \%$ \\
\hline 34 & plasma membrane part & GO:0044459 & 1918 & 104 & $5.42 \%$ \\
\hline 35 & cell fraction & GO:0000267 & 1039 & 55 & $5.29 \%$ \\
\hline 36 & cytoplasmic vesicle & GO:0031410 & 628 & 33 & $5.25 \%$ \\
\hline 37 & vesicle & GO:0031982 & 655 & 33 & $5.04 \%$ \\
\hline 38 & insoluble fraction & GO:0005626 & 803 & 34 & $4.23 \%$ \\
\hline 39 & plasma membrane & GO:0005886 & 3650 & 139 & $3.81 \%$ \\
\hline 40 & cytosol & GO:0005829 & 1251 & 47 & $3.76 \%$ \\
\hline Rank & MOLECULAR FUNCTION & GO ID & COUNT & Observed & RATIO \\
\hline 1 & arginine binding & GO:0034618 & 3 & 3 & $100.00 \%$ \\
\hline 2 & nitric-oxide synthase activity & GO:0004517 & 3 & 3 & $100.00 \%$ \\
\hline 3 & tetrahydrobiopterin binding & GO:0034617 & 3 & 3 & $100.00 \%$ \\
\hline 4 & C-X-C chemokine binding & GO:0019958 & 8 & 4 & $50.00 \%$ \\
\hline 5 & beta-amyloid binding & GO:0001540 & 13 & 5 & $38.46 \%$ \\
\hline 6 & tumor necrosis factor receptor binding & GO:0005164 & 21 & 8 & $38.10 \%$ \\
\hline
\end{tabular}


Table 1 Gene Ontology enrichment analysis (Continued)

\begin{tabular}{|c|c|c|c|c|c|}
\hline 7 & chemokine activity & GO:0008009 & 47 & 17 & $36.17 \%$ \\
\hline 8 & chemokine receptor binding & GO:0042379 & 49 & 17 & $34.69 \%$ \\
\hline 9 & coreceptor activity & GO:0015026 & 19 & 6 & $31.58 \%$ \\
\hline 10 & tumor necrosis factor receptor superfamily binding & GO:0032813 & 31 & 9 & $29.03 \%$ \\
\hline 11 & cytokine receptor binding & GO:0005126 & 178 & 46 & $25.84 \%$ \\
\hline 12 & chemokine binding & GO:0019956 & 26 & 6 & $23.08 \%$ \\
\hline 13 & cytokine activity & GO:0005125 & 196 & 45 & $22.96 \%$ \\
\hline 14 & growth factor receptor binding & GO:0070851 & 67 & 14 & $20.90 \%$ \\
\hline 15 & collagen binding & GO:0005518 & 35 & 7 & $20.00 \%$ \\
\hline 16 & G-protein-coupled receptor binding & GO:0001664 & 107 & 20 & $18.69 \%$ \\
\hline 17 & integrin binding & GO:0005178 & 58 & 9 & $15.52 \%$ \\
\hline 18 & cysteine-type endopeptidase activity & GO:0004197 & 71 & 10 & $14.08 \%$ \\
\hline 19 & growth factor activity & GO:0008083 & 161 & 19 & $11.80 \%$ \\
\hline 20 & cytokine binding & GO:0019955 & 108 & 12 & $11.11 \%$ \\
\hline 21 & protein heterodimerization activity & GO:0046982 & 189 & 21 & $11.11 \%$ \\
\hline 22 & glycosaminoglycan binding & GO:0005539 & 139 & 14 & $10.07 \%$ \\
\hline 23 & protein complex binding & GO:0032403 & 196 & 19 & $9.69 \%$ \\
\hline 24 & receptor binding & GO:0005102 & 856 & 83 & $9.70 \%$ \\
\hline 25 & receptor signaling protein activity & GO:0005057 & 159 & 15 & $9.43 \%$ \\
\hline 26 & pattern binding & GO:0001871 & 153 & 14 & $9.15 \%$ \\
\hline 27 & peptidase inhibitor activity & GO:0030414 & 154 & 14 & $9.09 \%$ \\
\hline 28 & carbohydrate binding & GO:0030246 & 349 & 29 & $8.31 \%$ \\
\hline 29 & endopeptidase activity & GO:0004175 & 370 & 28 & $7.57 \%$ \\
\hline 30 & polysaccharide binding & GO:0030247 & 153 & 14 & $9.15 \%$ \\
\hline 31 & protein dimerization activity & GO:0046983 & 514 & 36 & $7.00 \%$ \\
\hline 32 & identical protein binding & GO:0042802 & 618 & 38 & $6.15 \%$ \\
\hline 33 & enzyme binding & GO:0019899 & 505 & 29 & $5.74 \%$ \\
\hline 34 & peptidase activity & GO:0008233 & 563 & 30 & $5.33 \%$ \\
\hline 35 & peptidase activity, acting on L-amino acid peptides & GO:0070011 & 546 & 29 & $5.31 \%$ \\
\hline 36 & molecular transducer activity & GO:0060089 & 2116 & 98 & $4.63 \%$ \\
\hline 37 & signal transducer activity & GO:0004871 & 2116 & 98 & $4.63 \%$ \\
\hline 38 & receptor activity & GO:0004872 & 1674 & 71 & $4.24 \%$ \\
\hline 39 & protein binding & GO:0005515 & 8041 & 280 & $3.48 \%$ \\
\hline 40 & binding & GO:0005488 & 12465 & 320 & $2.57 \%$ \\
\hline
\end{tabular}

The table ranks the gene enrichment in biological processes, cellular component and molecular function with corresponding GO IDs. For each GO ID, the number of Observed Genes identified in the SSKB was divided by the number of Reference Genes in the human genome to calculate the Ratio of enrichment (Ratio).

showed highly significant enrichment $(\mathrm{P}<0.001)$ in this analysis (Table 2).

The pathway analysis revealed dominant pathways associated with immune regulation. Indeed, the eight most highly enriched pathways were associated with antigen presenting cells and activation of T cells and B cells.

Several cancer associated pathways were identified. This is partly due to the overlap between cancer pathways. These pathways typically include cytokine or growth factor stimulation of cell cycle and cell death and were not further analyzed.

Pathways associated with apoptosis, cytokine signaling and inflammation were also highly enriched. To focus on the events associated with initiation of Sjögren's syndrome, we analyzed pathways with known triggers. Several of the highly enriched pathways are triggered by bacterial toxins, viral DNA, or viral RNA. These include signaling pathways for Toll-like receptor, NOD-like receptor, RIG-I- 
Table 2 Biological pathways associated with SSKB genes

\begin{tabular}{|c|c|c|c|c|c|}
\hline Rank & PATHWAY & SSKB Genes & ENRICHMENT & Raw P & Adjust $\mathrm{P}$ \\
\hline 1 & Allograft rejection & 23 & 76.02 & $3.62 E-39$ & $6.82 \mathrm{E}-38$ \\
\hline 2 & Intestinal immune network for IgA production & 27 & 67.82 & $7.26 \mathrm{E}-44$ & $2.05 E-42$ \\
\hline 3 & Asthma & 14 & 58.61 & 4.14E-22 & $2.75 E-21$ \\
\hline 4 & Type I diabetes mellitus & 20 & 57.09 & $9.13 \mathrm{E}-31$ & $9.38 \mathrm{E}-30$ \\
\hline 5 & Graft-versus-host disease & 18 & 53.83 & $3.21 \mathrm{E}-27$ & $2.79 \mathrm{E}-26$ \\
\hline 6 & Autoimmune thyroid disease & 22 & 52.13 & $1.29 \mathrm{E}-32$ & $1.82 \mathrm{E}-31$ \\
\hline 7 & Primary immunodeficiency & 14 & 50.24 & $6.38 \mathrm{E}-21$ & $3.79 \mathrm{E}-20$ \\
\hline 8 & Hematopoietic cell lineage & 33 & 47.1 & $1.39 E-46$ & $5.24 \mathrm{E}-45$ \\
\hline 9 & Toll-like receptor signaling pathway & 37 & 46.01 & $1.13 \mathrm{E}-51$ & $6.38 \mathrm{E}-50$ \\
\hline 10 & Apoptosis & 25 & 35.68 & $5.55 E-32$ & $6.97 E-31$ \\
\hline 11 & NOD-like receptor signaling pathway & 17 & 34.44 & 7.61E-22 & 4.78E-21 \\
\hline 12 & Amyotrophic lateral sclerosis (ALS) & 14 & 33.18 & $5.81 \mathrm{E}-18$ & $2.85 \mathrm{E}-17$ \\
\hline 13 & Other glycan degradation & 4 & 31.4 & 6.67E-06 & $1.24 \mathrm{E}-05$ \\
\hline 14 & Cytokine-cytokine receptor interaction & 66 & 31.05 & 5.91E-79 & $6.68 \mathrm{E}-77$ \\
\hline 15 & T cell receptor signaling pathway & 26 & 30.24 & 4.12E-31 & 4.66E-30 \\
\hline 16 & RIG-I-like receptor signaling pathway & 17 & 30.07 & $9.98 \mathrm{E}-21$ & $5.64 \mathrm{E}-20$ \\
\hline 17 & Cell adhesion molecules (CAMs) & 32 & 29.99 & $6.40 E-38$ & $1.03 \mathrm{E}-36$ \\
\hline 18 & Bladder cancer & 10 & 29.9 & 1.06E-12 & $3.24 \mathrm{E}-12$ \\
\hline 19 & Viral myocarditis & 17 & 29.25 & $1.68 \mathrm{E}-20$ & $9.04 \mathrm{E}-20$ \\
\hline 20 & Cytosolic DNA-sensing pathway & 13 & 29.16 & $5.78 \mathrm{E}-16$ & $2.42 \mathrm{E}-15$ \\
\hline 21 & Pancreatic cancer & 15 & 26.17 & $1.88 \mathrm{E}-17$ & $8.50 \mathrm{E}-17$ \\
\hline 22 & Small cell lung cancer & 16 & 23.92 & 7.32E-18 & $3.45 \mathrm{E}-17$ \\
\hline 23 & Glycosaminoglycan degradation & 4 & 23.92 & $2.13 \mathrm{E}-05$ & $3.65 \mathrm{E}-05$ \\
\hline 24 & Natural killer cell mediated cytotoxicity & 25 & 22.92 & $1.06 \mathrm{E}-26$ & $8.56 \mathrm{E}-26$ \\
\hline 25 & ErbB signaling pathway & 13 & 22.16 & $2.51 \mathrm{E}-13$ & $8.86 \mathrm{E}-13$ \\
\hline 26 & Epithelial cell signaling in Helicobacter pylori infection & 12 & 22.16 & 2.64E-13 & $9.04 \mathrm{E}-13$ \\
\hline 27 & Complement and coagulation cascades & 12 & 21.84 & $3.17 \mathrm{E}-13$ & $1.05 \mathrm{E}-12$ \\
\hline 28 & B cell receptor signaling pathway & 13 & 21.77 & $3.38 \mathrm{E}-14$ & $1.23 \mathrm{E}-13$ \\
\hline 29 & Prion diseases & 6 & 21.53 & 3.27E-07 & $6.84 \mathrm{E}-07$ \\
\hline 30 & Antigen processing and presentation & 15 & 21.17 & 5.49E-16 & $2.39 \mathrm{E}-15$ \\
\hline 31 & Colorectal cancer & 14 & 20.93 & $6.14 \mathrm{E}-15$ & $2.48 \mathrm{E}-14$ \\
\hline 32 & Adipocytokine signaling pathway & 11 & 20.62 & $6.05 \mathrm{E}-12$ & $1.80 \mathrm{E}-11$ \\
\hline 33 & Chemokine signaling pathway & 30 & 19.83 & 7.80E-30 & 7.35E-29 \\
\hline 34 & Prostate cancer & 14 & 19.76 & $1.42 \mathrm{E}-14$ & $5.53 \mathrm{E}-14$ \\
\hline 35 & Glioma & 10 & 19.32 & 1.10E-10 & $2.89 \mathrm{E}-10$ \\
\hline 36 & Jak-STAT signaling pathway & 23 & 18.64 & 1.67E-22 & $1.18 \mathrm{E}-21$ \\
\hline 37 & Non-small cell lung cancer & 8 & 18.61 & 1.13E-08 & $2.50 \mathrm{E}-08$ \\
\hline 38 & Melanoma & 10 & 17.69 & 2.71E-10 & $6.96 \mathrm{E}-10$ \\
\hline 39 & Pathways in cancer & 46 & 17.51 & $9.85 \mathrm{E}-43$ & $2.23 \mathrm{E}-41$ \\
\hline 40 & Fc epsilon RI signaling pathway & 11 & 17.49 & $3.90 \mathrm{E}-11$ & $1.05 \mathrm{E}-10$ \\
\hline 41 & Chronic myeloid leukemia & 10 & 16.75 & 4.74E-10 & 1.19E-09 \\
\hline 42 & GnRH signaling pathway & 12 & 14.92 & $3.42 \mathrm{E}-11$ & $9.43 \mathrm{E}-11$ \\
\hline 43 & Leukocyte transendothelial migration & 14 & 14.9 & $7.91 \mathrm{E}-13$ & $2.48 \mathrm{E}-12$ \\
\hline 44 & VEGF signaling pathway & 9 & 14.87 & $1.04 \mathrm{E}-08$ & $2.35 \mathrm{E}-08$ \\
\hline
\end{tabular}


Table 2 Biological pathways associated with SSKB genes (Continued)

\begin{tabular}{|c|c|c|c|c|c|}
\hline 45 & Hypertrophic cardiomyopathy (HCM) & 10 & 14.78 & 1.67E-09 & 4.10E-09 \\
\hline 46 & p53 signaling pathway & 8 & 14.56 & 8.19E-08 & $1.75 \mathrm{E}-07$ \\
\hline 47 & Endometrial cancer & 6 & 14.49 & $3.65 \mathrm{E}-06$ & 7.11E-06 \\
\hline 48 & Systemic lupus erythematosus & 16 & 14.35 & $3.27 \mathrm{E}-14$ & $1.23 \mathrm{E}-13$ \\
\hline 49 & MAPK signaling pathway & 30 & 14.01 & $3.15 \mathrm{E}-25$ & 2.37E-24 \\
\hline 50 & Focal adhesion & 22 & 13.75 & $1.21 \mathrm{E}-18$ & $6.21 \mathrm{E}-18$ \\
\hline 51 & Dilated cardiomyopathy & 10 & 13.65 & $3.66 \mathrm{E}-09$ & 8.44E-09 \\
\hline 52 & Type II diabetes mellitus & 5 & 13.36 & $3.63 \mathrm{E}-05$ & $6.12 \mathrm{E}-05$ \\
\hline 53 & Neurotrophin signaling pathway & 13 & 12.96 & $3.17 \mathrm{E}-11$ & 8.96E-11 \\
\hline 54 & ECM-receptor interaction & 8 & 11.96 & $3.85 \mathrm{E}-07$ & 7.91E-07 \\
\hline 55 & Alzheimer's disease & 16 & 11.89 & $6.32 \mathrm{E}-13$ & 2.04E-12 \\
\hline 56 & Lysosome & 11 & 11.81 & $2.86 \mathrm{E}-09$ & $6.73 \mathrm{E}-09$ \\
\hline 57 & Arginine and proline metabolism & 5 & 11.63 & 7.15E-05 & 0.0001 \\
\hline 58 & Renal cell carcinoma & 6 & 10.77 & $2.09 \mathrm{E}-05$ & 3.63E-05 \\
\hline 59 & Long-term depression & 6 & 10.77 & $2.09 \mathrm{E}-05$ & 3.63E-05 \\
\hline 60 & Long-term potentiation & 6 & 10.77 & 2.09E-05 & 3.63E-05 \\
\hline 61 & Proteasome & 4 & 10.47 & 0.0006 & 0.0009 \\
\hline 62 & Progesterone-mediated oocyte maturation & 7 & 10.22 & $6.00 \mathrm{E}-06$ & 1.15E-05 \\
\hline 63 & TGF-beta signaling pathway & 7 & 10.11 & $6.48 \mathrm{E}-06$ & $1.22 \mathrm{E}-05$ \\
\hline 64 & Regulation of actin cytoskeleton & 16 & 9.3 & $2.69 \mathrm{E}-11$ & $7.79 \mathrm{E}-11$ \\
\hline 65 & Calcium signaling pathway & 13 & 9.17 & 2.36E-09 & 5.67E-09 \\
\hline 66 & Wnt signaling pathway & 11 & 9.15 & 4.17E-08 & 9.06E-08 \\
\hline 67 & Gap junction & 6 & 8.37 & 8.67E-05 & 0.0001 \\
\hline 68 & Cell cycle & 8 & 7.85 & $9.32 \mathrm{E}-06$ & 1.70E-05 \\
\hline 69 & Oocyte meiosis & 7 & 7.71 & $3.80 \mathrm{E}-05$ & $6.31 \mathrm{E}-05$ \\
\hline 70 & Axon guidance & 7 & 6.82 & 8.33E-05 & 0.0001 \\
\hline 71 & Endocytosis & 10 & 6.72 & $2.93 \mathrm{E}-06$ & 5.81E-06 \\
\hline 72 & Metabolic pathways & 26 & 2.96 & $1.12 \mathrm{E}-06$ & 2.26E-06 \\
\hline
\end{tabular}

The table lists the number of SSKB genes associated with individual KEGG pathways. The pathways are ranked according to their Enrichment relative to the number of reference genes in the human genome based on the hypergeometric test. The raw $P$-values (hypergeometric test) and the multiple test-adjusted $P$-values are listed for each pathway.

like receptor signaling pathways and the cytosolic DNAsensing pathway.

\section{Overlap with other autoimmune diseases}

The KEGG pathways include several pathways for autoimmune diseases, including type I diabetes mellitus, autoimmune thyroid disease, and SLE. While about 50\% of the genes associated with the first two pathways are also associated with Sjögren's syndrome, only 16 Sjögren's syndrome genes were identified in the 140-gene SLE pathway (KEGG ID: hsa05322). These findings suggest that significant differences exist in the pathogenesis of autoimmune diseases.

\section{Conclusions}

The results of this analysis can serve as a background and comparison for the increasing number of gene expression data sets available for Sjögren's syndrome, e.g. [15-17]. Preliminary analysis of such data sets suggest that the biological pathways identified in the SSKB are very similar to those identified in human parotid tissue but quite different from those identified in human labial salivary glands [15]. Future analyses will further define these differences and focus on the comparison of biological pathways identified in human tissues and mouse models of Sjögren's syndrome. It is envisioned that the SSKB data can also serve as the starting point for literature reviews and literature-based validation of identified genes; functional gene enrichment studies; protein-protein interaction networks and other bioinformatics analyses; it can be used to arrive at gene sets for SNP set enrichment analysis (pathway based GWAS studies); it can be used to define a gene set for gene set enrichment analysis (GSEA); as a starting point for bioinformatics analysis 
protein-protein interaction networks (based on yeast 2 hybrid) can be identified among the SSKB genes.

\section{Availability and requirements}

The Sjögren's syndrome knowledge base is freely available at sskb.umn.edu.

\section{Competing interests}

Dr. David Wong is scientific advisor to RNAmeTRIX Inc, a molecular diagnostics company. The authors declare no conflicts of interest.

\section{Authors' contributions}

SUG collected and organized data and performed data analysis and drafted the manuscript. TW designed and implemented the database and web site. DTWW contributed to data analysis, critical review of the database and editing of the manuscript. SH contributed to critical review of the database, statistical analysis and editing the manuscript. SM contributed to data analysis, critical review of the database and drafting the manuscript. Al authors read and approved the final manuscript.

\section{Acknowledgements}

The authors thank Dr. Ammon Peck, University of Florida Dr. Michael Zhou, UCLA for helpful discussions. The Minnesota Supercomputing Institute provides web hosting for the SSKB database. This work was supported by U. S. PHS grants R01DE019255 (SUG, SM, DTW) and R01DE014385 (SM) from NIDCR and a research grant from the Sjögren's Syndrome Foundation (SM).

\section{Author details}

${ }^{1}$ Department of Diagnostic and Biological Sciences, University of Minnesota School of Dentistry, Minneapolis, MN 55455, USA. ${ }^{2}$ Minnesota Supercomputing Institute, University of Minnesota, Minneapolis, MN 55455, USA. ${ }^{3}$ Department of Biostatistics, School of Public Health, University of California, Los Angeles, CA 90095, USA. ${ }^{4}$ School of Dentistry, University of California, Los Angeles, CA 90095, USA. ${ }^{5}$ Department of Pathology, Stanford University School of Medicine, Stanford, CA 94305, USA

Received: 10 October 2011 Accepted: 18 June 2012

Published: 3 July 2012

\section{References}

1. Meijer JM, Meiners PM, Huddleston Slater JJ, Spijkervet FK, Kallenberg CG, Vissink A, Bootsma H: Health-related quality of life, employment and disability in patients with Sjogren's syndrome. Rheumatology 2009, 48(9):1077-1082.

2. Rhodus NL: Sjogren's syndrome. Quintessence Int 1999, 30(10):689-699.

3. Williams PH, Cobb BL, Namjou B, Scofield RH, Sawalha AH, Harley JB: Horizons in Sjogren's syndrome genetics. Clin Rev Allergy Immunol 2007, 32(3):201-209.

4. Ercolini AM, Miller SD: The role of infections in autoimmune disease. Clin Exp Immunol 2009, 155(1):1-15.

5. Mavragani $\mathrm{CP}$, Crow MK: Activation of the type I interferon pathway in primary Sjogren's syndrome. J Autoimmun 2010, 35(3):225-231.

6. Peck AB, Nguyen CQ, Sharma A, Mclndoe RA, She JX: The Interferon-Signature of Sjögren's Syndrome: What does It say about the etiopathology of autoimmunity? J Clin Rheumatol Musculoskel Med 2011, 3(1).

7. Rosen A, Casciola-Rosen L: Altered autoantigen structure in Sjogren's syndrome: implications for the pathogenesis of autoimmune tissue damage. Crit Rev Oral Biol Med 2004, 15(3):156-164.

8. Stathopoulou EA, Routsias JG, Stea EA, Moutsopoulos HM, Tzioufas AG: Cross-reaction between antibodies to the major epitope of Ro60 kD autoantigen and a homologous peptide of Coxsackie virus 2B protein. Clin Exp Immunol 2005, 141(1):148-154.

9. Zhao Z-S, Granucci F, Yeh L, Schaffer PA, Cantor H: Molecular mimicry by herpes simplex virus-type 1: autoimmune disease after viral infection. Science 1998, 279(5355):1344-1347.

10. Nguyen KH, Brayer J, Cha S, Diggs S, Yasunari U, Hilal G, Peck AB, Humphreys-Beher MG: Evidence for antimuscarinic acetylcholine receptor antibody-mediated secretory dysfunction in NOD mice. Arthritis Rheum 2000, 43(10):2297-2306.
11. Li J, Ha Y, Ku N, Choi S, Lee S, Oh S, Kim J, Lee J, Lee E, Song Y, et al: Inhibitory effects of autoantibodies on the muscarinic receptors in Sjogren's syndrome. Lab Invest 2004, 84(11):1430-1438.

12. von Bultzingslöwen I, Sollecito TP, Fox PC, Daniels T, Jonsson R, Lockhart PB, Wray D, Brennan MT, Carrozzo M, Gandera B, et al: Salivary dysfunction associated with systemic diseases: systematic review and clinical management recommendations. Oral Surg Oral Med Oral Pathol Oral Radiol Endod 2007, 103(Suppl):S75.e1-e15.

13. Meijer JM, Meiners PM, Vissink A, Spijkervet FK, Abdulahad W, Kamminga N, Brouwer E, Kallenberg CG, Bootsma H: Effectiveness of rituximab treatment in primary Sjogren's syndrome: a randomized, double-blind, placebo-controlled trial. Arthritis Rheum 2010, 62(4):960-968.

14. Moutsopoulos NM, Katsifis GE, Angelov N, Leakan RA, Sankar V, Pillemer S, Wahl SM: Lack of efficacy of etanercept in Sjogren's syndrome correlates with failed suppression of TNF\{alpha\} and systemic immune activation. Ann Rheum Dis 2008, 67(10):1437-1443.

15. Gottenberg J-E, Cagnard N, Lucchesi C, Letourneur F, Mistou S, Lazure T, Jacques S, Ba N, Ittah M, Lepajolec C, et al: Activation of IFN pathways and plasmacytoid dendritic cell recruitment in target organs of primary Sjogren's syndrome. Proc Natl Acad Sci U S A 2006, 103(8):2770-2775

16. Hu S, Zhou M, Jiang J, Wang J, Elashoff D, Gorr S, Michie SA, Spijkervet FK, Bootsma H, Kallenberg CG, et al: Systems biology analysis of Sjogren's syndrome and mucosa-associated lymphoid tissue lymphoma in parotid glands. Arthritis Rheum 2009, 60(1):81-92.

17. Nguyen CQ, Sharma A, Lee BH, She JX, Mclndoe RA, Peck AB: Differential gene expression in the salivary gland during development and onset of xerostomia in Sjogren's syndrome-like disease of the C57BL/6.NODAec1Aec2 mouse. Arthritis Res Ther 2009, 11(2):R56.

18. Sjogren's Syndrome Knowledge Base. http://sskb.umn.edu/.

19. PubMed. http://www.ncbi.nlm.nih.gov/pubmed/.

20. Rebholz-Schuhmann D, Kirsch H, Arregui M, Gaudan S, Riethoven M, Stoehr P: EBIMed - text crunching to gather facts for proteins from Medline. Bioinformatics 2007, 23(2):e237-e244.

21. Entrez. http://www.ncbi.n/m.nih.gov/Entrez/.

22. UniProt. http://www.uniprot.org/.

23. Consortium TU: Ongoing and future developments at the Universal Protein Resource. Nucleic Acids Res 2011, 39(suppl 1):D214-D219.

24. WebGestalt. http://bioinfo.vanderbilt.edu/webgestalt/.

25. Zhang B, Kirov S, Snoddy J: WebGestalt: an integrated system for exploring gene sets in various biological contexts. Nucleic acids research 2005, 33(Web Server issue):W741-W748.

26. Benjamini $Y$, Hochberg $Y$ : Controlling the false discovery rate - a practical and powerful approach to multiple testing. J Roy Stat Soc B Met 1995, 57(1):289-300.

27. The Gene Ontology. http://www.geneontology.org/.

28. Ashburner M, Ball CA, Blake JA, Botstein D, Butler H, Cherry JM, Davis AP, Dolinski K, Dwight SS, Eppig JT, et al: Gene ontology: tool for the unification of biology. The Gene Ontology Consortium. Nat Genet 2000, 25(1):25-29

29. Kyoto Encyclopedia of Genes and Genomes. http://www.genome.jp/kegg/

30. Kanehisa M, Goto S: KEGG: Kyoto Encyclopedia of Genes and Genomes. Nucleic Acids Res 2000, 28(1):27-30.

31. Nguyen $C Q$, Peck $A B$ : Unraveling the pathophysiology of Sjogren syndrome-associated dry eye disease. Ocul Surf 2009, 7(1):11-27.

32. Mariette $X$, Gottenberg JE: Pathogenesis of Sjogren's syndrome and therapeutic consequences. Curr Opin Rheumatol 2010, 22 (5):471-477.

33. Sall K, Stevenson OD, Mundorf TK, Reis BL: Two multicenter, randomized studies of the efficacy and safety of cyclosporine ophthalmic emulsion in moderate to severe dry eye disease. CsA Phase 3 Study Group. Ophthalmology 2000, 107(4):631-639.

34. Oxholm P, Winther K, Manthorpe R: Platelets in blood and salivary glands of patients with primary Sjogren's syndrome. Scand J Rheumatol Suppl 1986, 61:170-172.

35. Lodde BM, Sankar V, Kok MR, Leakan RA, Tak PP, Pillemer SR: Serum lipid levels in Sjögren's syndrome. Rheumatology 2006, 45(4):481-484.

36. Ostrowski RA, Robinson JA: Antiphospholipid antibody syndrome and autoimmune diseases. Hematol Oncol Clin North Am 2008, 22(1):53-65. 
37. Chai J, Logigian EL: Neurological manifestations of primary Sjogren's syndrome. Curr Opin Neurol 2010, 23(5):509-513.

38. Caulfield $\mathrm{VL}$, Balmer C, Dawson LJ, Smith PM: A role for nitric oxide-mediated glandular hypofunction in a non-apoptotic model for Sjogren's syndrome. Rheumatology 2009, 48(7):727-733.

doi:10.1186/1471-2474-13-119

Cite this article as: Gorr et al:: Text-mining applied to autoimmune disease research: the Sjögren's syndrome knowledge base. BMC

Musculoskeletal Disorders 2012 13:119.

\section{Submit your next manuscript to BioMed Central and take full advantage of:}

- Convenient online submission

- Thorough peer review

- No space constraints or color figure charges

- Immediate publication on acceptance

- Inclusion in PubMed, CAS, Scopus and Google Scholar

- Research which is freely available for redistribution 